\title{
Histological validation of dynamic-equilibrium cardiovascular magnetic resonance for the measurement of myocardial extracellular volume
}

\author{
Christopher A Miller ${ }^{1,2 *}$, Josephine H Naish², Paul Bishop ${ }^{3}$, Glyn Coutts ${ }^{5}$, David Clark ${ }^{4}$, Sha Zhao ${ }^{2}$, Simon G Ray ${ }^{1}$, \\ Nizar Yonan ${ }^{1}$, Simon G Williams ${ }^{1}$, Andrew Flett ${ }^{6,7}$, James Moon ${ }^{6,7}$, Geoffrey Parker ${ }^{2}$, Matthias Schmitt ${ }^{1,2}$
}

From 16th Annual SCMR Scientific Sessions

San Francisco, CA, USA. 31 January - 3 February 2013

\section{Background}

Extracellular matrix expansion is fundamental to left ventricular (LV) remodeling, and is a therapeutic target. CMR techniques are increasingly used to evaluate myocardial extracellular volume (ECV), however the most widely applied methods are without histological validation. The aim of this study was to provide whole-heart, histological validation of; 1. Dynamic-equilibrium CMR (DynEq-CMR), where ECV is quantified using hematocrit-adjusted myocardial and blood $\mathrm{T} 1$ values measured before and after gadolinium bolus; and 2. Isolated measurement of myocardial $\mathrm{T} 1$ at a fixed time-point following gadolinium bolus, used as an ECV surrogate.

\section{Methods}

CMR was performed prospectively in patients awaiting heart transplantation. Of 54 patients on the transplant waiting list at a single UK Center between Jan 12011 and July 1 2012, 41 had contraindications to CMR, 2 were too unwell and 2 refused consent. The remaining 9 underwent CMR, including modified look locker inversion recovery imaging at base, mid and apical LV levels before and 10 and $15 \mathrm{mins}$ after $0.2 \mathrm{mmol} / \mathrm{Kg}$ Gd-DTPA bolus at $1.5 \mathrm{~T}$, and same-day hematocrit measurement. Resulting pixelwise T1 maps (MatLab) were used to calculate segmental ECV. (Phantom studies performed prior to patient scanning determined $\mathrm{T} 1$ measurement accuracy and heart-rate correction algorithm). 6 patients subsequently underwent transplantation (median interval between CMR and transplant 29 days). 16 tissue samples taken from each heart according to the 16-segment model (96 segments in total) were analysed for picrosirius red collagen volume fraction (CVF, Figure 1). The same CMR protocol was also performed in 10 matched healthy subjects.

\section{Results}

DynEq-CMR-derived ECV was linearly related to histological CVF $(\mathrm{p}<0.01$; within-subject $\mathrm{r}=0.75, \mathrm{p}<0.01$; $\mathrm{r} 2=0.56$; between-subject $\mathrm{r}=0.95, \mathrm{p}<0.01, \mathrm{r} 2=0.89$; for ECV calculated using $15 \mathrm{~min}$ post-contrast $\mathrm{T} 1$; linear regression equation: histological $\mathrm{CVF}=1.45 \mathrm{xECV}-42$, Figure 2). Correlation was maintained throughout the entire heart (i.e. across all ventricular levels and septal and non-septal segments), and when segments displaying late gadolinium enhancement (LGE) were included and excluded. Isolated post-contrast T1 measurement showed significant within-subject correlation with histological CVF $(r=-0.74, \mathrm{p}<0.01 ; \mathrm{r} 2=0.55$ for $15 \mathrm{~min}$ post-contrast T1), but between-subject correlations were not significant, likely reflecting between subject confounding factors such as renal function and body habitus. Pre-contrast T1 values and histological CVF were not significantly correlated. Mean segmental ECV in segments without LGE $(41.4 \pm 5.0 \%)$ and with LGE $(47.0$ $\pm 7.4 \%)$ were both significantly higher than in healthy subjects $(25.5 \pm 2.6 \% ; \mathrm{p}<0.01)$.

\section{Conclusions}

DynEq-CMR-derived ECV shows a good correlation with histological CVF throughout the whole heart. Isolated post-contrast T1 measurement is insufficient for ECV assessment. 


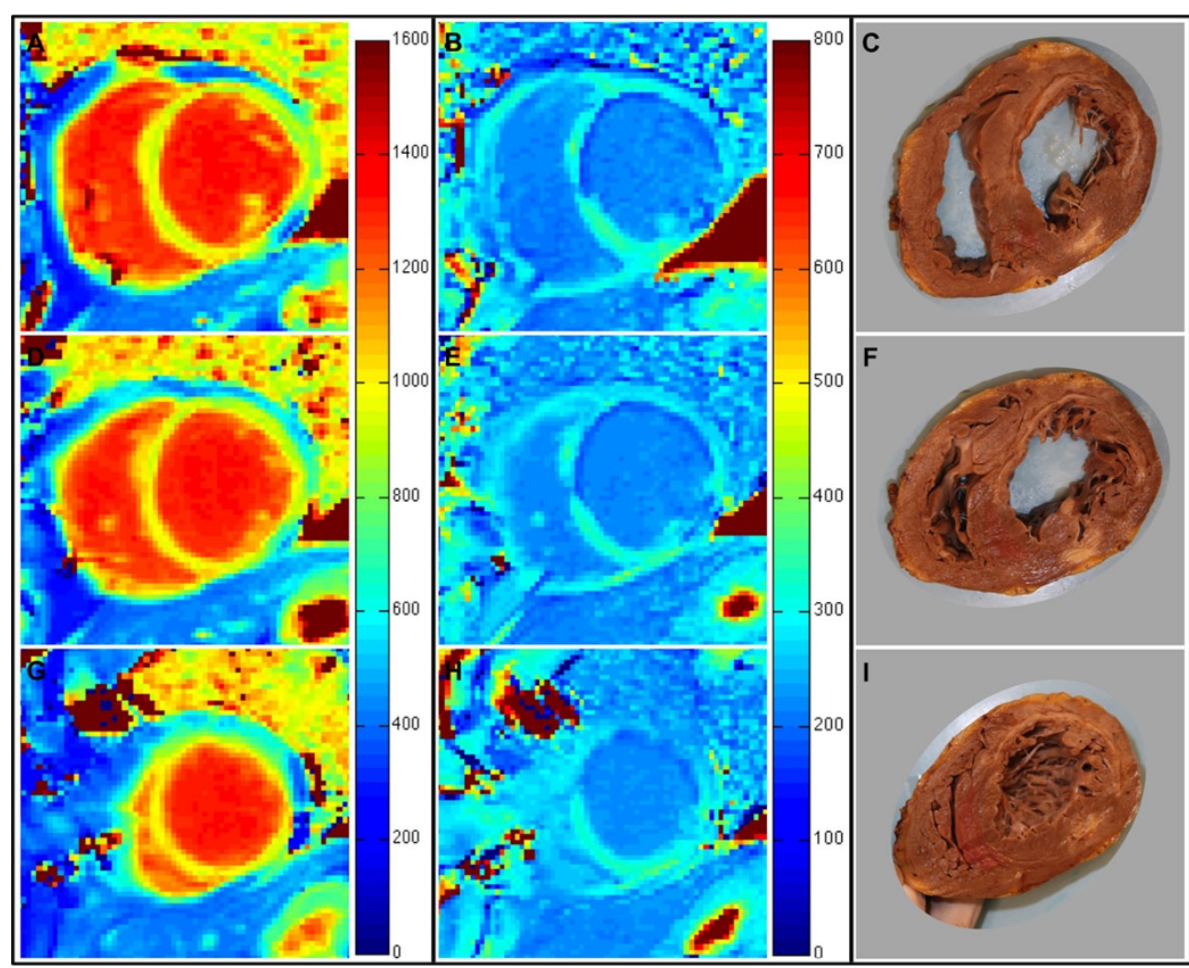

Figure 1 Histological validation of dynamic-equilibrium CMR. Short-axis pixelwise T1 relaxation time maps at basal (A and B; colors correspond to $T 1$ relaxation time according to colour bar legends), mid ( $D$ and $E$ ) and apical ( $G$ and $H$ ) ventricular levels before ( $A, D, G)$ and 15-minutes after a bolus of $0.20 \mathrm{mmol} / \mathrm{Kg} \mathrm{Gd}$-DTPA contrast $(B, E, H)$, were determined from modified Look Locker inversion recovery imaging in order to determine segmental myocardial extracellular volume fraction. Subsequently, patients underwent heart transplantation. Explanted hearts were immediately fixed in 10\% buffered formalin and cut at corresponding basal (C), mid (F) and apical (I) ventricular levels. Tissue blocks were taken from each segment before being embedded in paraffin and stained with picrosirius red in order to determine segmental histological collagen volume fraction.

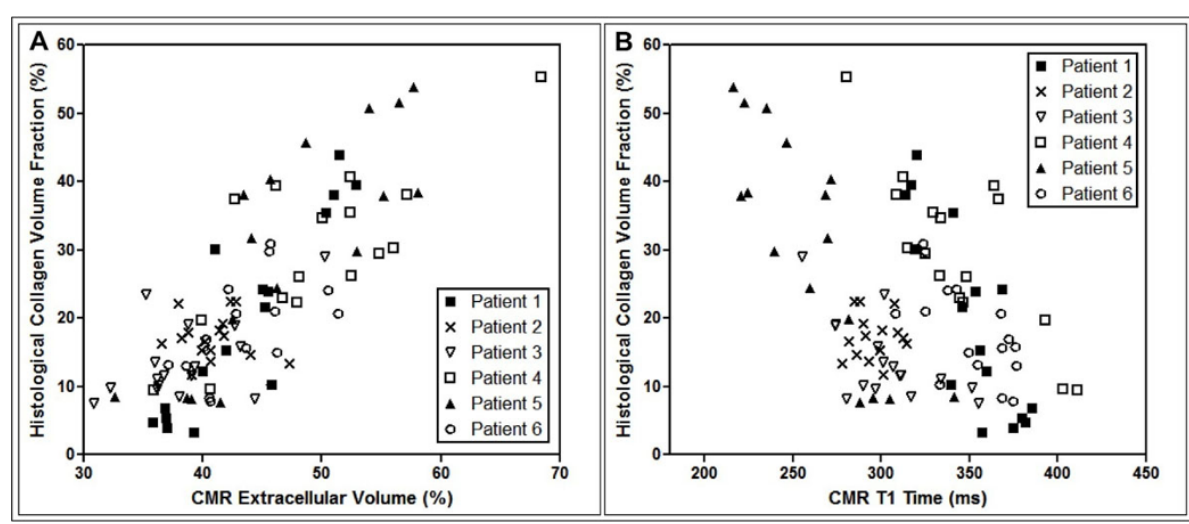

Figure 2 (A) Dynamic-equilibrium CMR-measured myocardial extracellular volume plotted against histological collagen volume fraction. (B) Isolated post-contrast T1 measurements made at 15-minutes post-contrast plotted against histological collagen volume fraction. Symbols correspond to different patients, as set out in the legends.

\section{Funding}

Christopher Miller is supported by a Doctoral Research Fellowship from the National Institute of Health Research (UK).

\section{Author details}

${ }^{1}$ North West Heart Centre and Transplant Unit, University Hospital of South Manchester, Manchester, UK. ${ }^{2}$ Biomedical Imaging Institute, University of Manchester, Manchester, UK. ${ }^{3}$ Department of Pathology, University Hospital of South Manchester, Manchester, UK. ${ }^{4}$ Alliance Cardiac MRI Unit, University Hospital of South Manchester, Manchester, UK. ${ }^{5}$ Christie Medical Physics and 
Engineering, The Christie Hospital, Manchester, UK. ${ }^{6}$ The Heart Hospital, London, UK. ${ }^{7}$ Institute of Cardiovascular Science, University College London, London, UK

Published: 30 January 2013

doi:10.1186/1532-429X-15-S1-016

Cite this article as: Miller et al:: Histological validation of dynamic-

equilibrium cardiovascular magnetic resonance for the measurement of myocardial extracellular volume. Journal of Cardiovascular Magnetic Resonance 2013 15(Suppl 1):016.

Submit your next manuscript to BioMed Central and take full advantage of:

- Convenient online submission

- Thorough peer review

- No space constraints or color figure charges

- Immediate publication on acceptance

- Inclusion in PubMed, CAS, Scopus and Google Scholar

- Research which is freely available for redistribution

Submit your manuscript at www.biomedcentral.com/submit
C Biomed Central 